\title{
Apego e parentalidade sob o enfoque transcultural: uma revisão da literatura
}

\author{
Attachment behavioral and parenting definitions, based on a \\ cross-cultural approach: a review of the literature
}

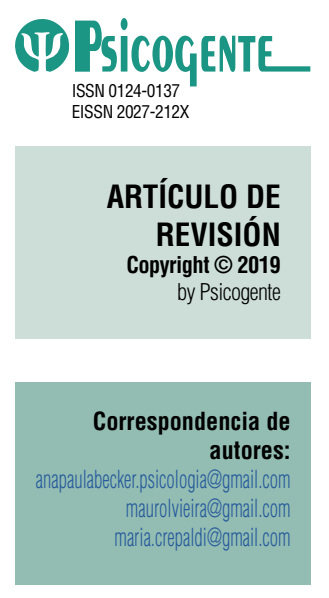

Recibido: 04-04-18 Aceptado: 08-04-19 Publicado: 01-07-19

\section{Apego y parentalidad desde el enfoque transcultural: una revisión de la literatura}

Ana Paula Sesti Becker (D) - Mauro Luis Vieira (DD - Maria Aparecida Crepaldi (iD) Universidade Federal de Santa Catarina (UFSC), Florianópolis, Brasil

Resumo

Objetivo: Analisar sistematicamente a produção científica acerca do apego e a parentalidade sob o enfoque transcultural.

Método: Realizou-se o levantamento de produções indexadas, a partir de buscas em bases de dados nacionais -Scielo Brasil- e CAPES - Coordenação de Aperfeiçoamento de Pessoal de Nível Superior-; e internacionais -PsycINFO-, mediante os seguintes descritores: Apego OR Bowlby AND parentalidade OR "relação parental"; Attachment OR Bowlby AND Parenting OR Parenthood; e por fim, Apego OR Bowlby AND Parentalidad OR "Relaciones parentales". Foram considerados os estudos nos idiomas português, inglês e espanhol, entre o período de 2013 a outubro de 2017. Após a definição dos critérios de inclusão, os cinco artigos retidos foram analisados com base nos aspectos metodológicos (dados da amostra, tipo de abordagem, tipo de estudo e técnica de coleta de dados), e posteriormente, foram submetidos a análise semântica, da qual derivou-se duas categorias temáticas: 1) Fatores que influenciam a formação do apego e a parentalidade e 2) Apego e relações parentais.

Resultados: Apontaram-se lacunas existentes no que se refere à produção científica especificamente ao tema elencado; tendo em vista a diversidade de estudos que englobam os sistemas de apego e temas transversais, como apego e desenvolvimento infantil, apego e psicopatologia, apego e maternidade, entre outros. Além disso, não foram encontrados estudos nacionais sobre a temática, assim como pesquisas transculturais a respeito. Em relação ao delineamento dos estudos encontrados, todos caracterizam-se pelo enfoque quantitativo e estudos longitudinais do tipo prospectivos-follow-up. As técnicas de coleta de dados que mais se sobressaíram foram a Entrevista de Apego Adulto (AAI), seguida da Situação Estranha em pesquisas com amostras infantis.

Conclusão: Destaca-se que a Teoria do Apego necessita estar aberta para a revisão e a consideração das dimensões relacionadas à cultura. Isso implica ajustá-la à diferentes contextos culturais, históricos e sociais. Sugere-se, portanto, o incremento de novos estudos que contemplem a formação do apego, os quais venham disseminar intervenções parentais positivas para o ciclo de vida pessoal e familiar dos sujeitos.

Palavras-chave: apego, parentalidade, psicologia transcultural.

Abstract

Objective: This paper aims to analyze systematically the scientific production related to attachment behavioral and parenting based on a cross-cultural approach.

Method: A survey of indexed productions based on national databases such as: Scielo Brasil and CAPES -Coordination of Improvement of Higher Level Personnel-; and international -PsycINFO-, using the following descriptors: Apego OR Bowlby AND parenting OR "parental relationship"; Attachment OR Bowlby AND Parenting OR Parenthood; and finally, Apego OR Bowlby AND Parentality OR "Parental relationships"

Cómo citar este artículo (APA):

Sesti Becker, A. P., Vieira, M. L,. \& Aparecida Crepaldi, M. (2019). Apego e parentalidade sob o enfoque transcultural: uma revisão da literatura. Psicogente 22(42), 1-25. https://doi.org/10.17081/psico.22.42.3507 
was performed. Studies translated into Portuguese, English and Spanish between 2013 and October 2017 were considered. After defining the inclusion criteria, the five retained articles were analyzed based on the methodological aspects (sample data, type of approach, type of study and data collection technique), and afterwards, they were submitted to semantic analysis, from which we derived two thematic categories: 1) Factors that influence the formation of attachment and parenting, and 2) Attachment and parental relationships.

Results: Gaps corresponding to the scientific production according to the topics suggested were identified; identifying the diversity of studies related to attachment behavioral systems and cross-cutting themes, such as: child attachment behavioral and development, attachment behavioral and psychopathology, attachment behavioral and motherhood, among others, were taken into account. In addition, there is no national studies based on these topics, as well as cross-cultural surveys. A quantitative approach and prospective longitudinal studies (follow-up) were conducted in these studies. In order to collect the data, Adult Attachment Interview (AAI) followed by The Strange Situation Survey used with children sampled, were highlighted.

Conclusion: It should be noted that the Theory of Attachment needs to be open to the review and consideration of dimensions related to culture. This implies adjusting it to different cultural, historical and social contexts. It is suggested, therefore, the increase of new studies that contemplate the formation of attachment, which will disseminate positive parental interventions for the individuals' personal and family life cycle.

Keywords: attachment, parenting, cross-cultural, phychology

Resumen

Objetivo: Analizar sistemáticamente la producción científica acerca del apego y la parentalidad bajo el enfoque transcultural.

Método: Se realizó el levantamiento de producciones indexadas a partir de búsquedas en bases de datos nacionales -Scielo Brasil- y CAPES -Coordinación de Perfeccionamiento de Personal de Nivel Superior-; e internacionales - PsycINFO-, mediante los siguientes descriptores: Apego OR Bowlby AND parentalidad OR "relación parental". Y por último, Apego OR Bowlby AND Parentalidad OR "Relaciones parentales". Se consideraron los estudios en portugués, inglés y español, entre el período de 2013 a octubre de 2017. Después de la definición de los criterios de inclusión, los cinco artículos retenidos se analizaron sobre la base de los aspectos metodológicos (datos de la muestra, tipo de enfoque, tipo de estudio y técnica de recolección de datos), y posteriormente se sometieron al análisis semántico; se derivaron dos categorías temáticas: 1) Factores que influencian la formación del apego y la parentalidad, y 2) Apego y relaciones parentales.

Resultados: Se señalaron las lagunas existentes en lo que se refiere a la producción científica específicamente al tema enumerado, identificando la diversidad de estudios que engloban los sistemas de apego y temas transversales, como apego y desarrollo infantil, apego y psicopatología, apego y maternidad, entre otros. Además, no se encontraron estudios nacionales sobre la temática, así como investigaciones transculturales al respecto. En relación al delineamiento de los estudios encontrados, todos se caracterizan por el enfoque cuantitativo y estudios longitudinales del tipo prospectivo follow-up. Las técnicas de recolección de datos que más sobresalieron fueron la Entrevista de Apego Adulto (AAI), seguida de la Situación Extraña en investigaciones con muestras infantiles.

Conclusión: Se destaca que la Teoría del Apego necesita estar abierta para la revisión y la consideración de las dimensiones relacionadas a la cultura. Esto implica ajustarla a diferentes contextos culturales, históricos y sociales. Se sugiere, por lo tanto, el incremento de nuevos estudios que contemplen la formación del apego, los cuales vengan a diseminar intervenciones parentales positivas para el ciclo de vida personal y familiar de los sujetos.

Palabras clave: apego, parentalidad, psicología transcultural.

\section{INTRODUÇÃO}

Desde o início das postulações da Teoria do Apego, diversas investigações têm sido levantadas dentro das temáticas que norteiam a Psicologia do Desenvolvimento (Pontes, Silva, Garotti \& Magalhães, 2007), além de consolidar-se como uma teoria clássica que traz importantes contribuições sobre a 
vinculação e afetividade humana (Melchiori \& Dessen, 2008; Pereira, Ferreira, \& Paredes, 2012). Destaca-se a ênfase atribuída aos aspectos teóricos do apego, os quais suscitam diferentes perspectivas de bases biológicas e culturalistas, como também confere um aporte para o manejo clínico, educacional e diversos programas de intervenção (Bowlby, 1988; Byng-Hall, 1995; Pontes et al., 2007; Bolsoni-Silva, Rodrigues, Abramides \& Souza, 2007; Cia, Pamplin \& Williams, 2008; Murta, Rodrigues, Rosa \& Paulo, 2011).

Embora a Teoria do Apego ainda seja recente, torna-se necessário delimitar os pontos fundamentais que legitimam sua origem e, posteriormente, as investigações contemporâneas que trazem novos olhares e questionamentos à teoria. Como aspectos fundamentais, pode-se dizer que o conceito de sensibilidade materna para o desenvolvimento dos padrões de apego e a transgeracionalidade da díade mãe-bebê, ainda permanece central nessa vertente teórica (Melchiori, \& Dessen, 2008).

Todavia, com o avanço do conhecimento na área e com base nas mudanças socioculturais, especialmente a partir da década de 1980, derivaram-se temáticas contemporâneas embasadas pela teoria sob diferentes enfoques, tais como: apego na adolescência e fase adulta (Johnson \& Whifen, 2012); apego e envolvimento paterno (Scola \& Vauclair, 2010; Newland \& Coyl, 2010; Backes, 2018); apego e vínculos conjugais (Cowan \& Cowan, 2016; Juric, 2009; Hazan \& Shaver, 1994); apego e luto (Parkes, 2009; Hauser, 2007); apego e a relação professor-aluno (Reio Jr., Marcus \& Sanders-Reio, 2009), entre outros.

De forma breve, oportuniza-se resgatar alguns eixos centrais que norteiam a epistemologia referida e, posteriormente, tecer algumas reflexões quanto ao apego e parentalidade sob a perspectiva transcultural, seguido da discussão de uma revisão de literatura sistemática com base na temática.

\subsection{Teoria do Apego e a parentalidade}

John Bowlby (1907-1991), médico Psiquiatra e Psicanalista britânico, foi o precursor da Teoria do Apego, em meados da segunda metade do século $X X$. A premissa fundamental que sustenta sua teoria, baseia-se na concepção de que os seres humanos apresentam uma inclinação natural primária para construir vínculos afetivos. Isso pode ser explicado pela condição de extrema vulnerabilidade fisiológica que os bebês apresentam quando vêm ao mundo, de modo que dependam de alguém que lhes garantam os cuidados básicos para a sua sobrevivência. Segundo Bowlby (1988), o apego é um tipo de vínculo afetivo biologicamente inato entre os seres humanos que visa à 
busca pela proximidade com outro indivíduo - figura ou objeto de apego, identificado como alguém disponível que oferece respostas e proporciona um sentimento de segurança, sendo considerado como mais preparado para lidar com o mundo.

Logo, o pressuposto básico da teoria é norteado pela qualidade da interação entre a criança e o cuidador primário. Nos anos iniciais de vida, a reciprocidade dos pais para atender às necessidades do filho deve favorecer o desenvolvimento de um senso de segurança, uma condição de bem-estar e confiança que servirá como base para a exploração do ambiente e do conhecimento, assim como do rendimento acadêmico (Gomes \& Bosa, 2010; Schmidt \& Argimon, 2009; Meza, Salazar \& Berrío, 2018). Arcón e Sánchez (2018) complementam que uma dinâmica relacional positiva entre pais e filhos caracteriza um dos fatores de proteção importantes para o desenvolvimento psicoafetivo das crianças.

Portanto, é nessa fase que se desenvolve o que Bowlby denominou como "modelo funcional do eu" ou mais recentemente por "representações mentais" e "script de apego", termos atualizados por alguns pesquisadores contemporâneos (Crowell \& Treboux, 2001; Waters \& Waters, 2006; Byng-Hall, 1995), por contemplar as representações internas da pessoa a respeito do mundo, as quais permitem incluir expectativas, crenças e imagens mentais sobre diversos aspectos da vida.

À medida que tais representações são desenvolvidas na criança, esta ao se tornar adulta, estabelecerá novas relações tendo por base o modelo formado em suas primeiras vinculações afetivas; deste modo, as diversas funções da vida adulta desempenhadas na vida conjugal, profissional e parental, serão influenciadas pelo apego estabelecido com a família de origem (Nascimento, \& Coelho, 2006; Bolze, 2016; Backes, 2018; Carter \& McGoldrick, 1995; McGoldrick \& Ashton, 2016).

No estudo realizado por Murta e cols (2011), o qual descreve uma avaliação de necessidades para o desenvolvimento de um programa de transição para a parentalidade, verificou-se a importância de investigar a experiência de apego do casal com seus progenitores em relação aos cuidados com seus filhos recém-nascidos. Como resultado foi apontado que os membros do casal que vivenciaram práticas parentais coercitivas na infância e apego inseguro podem apresentar maiores fatores de risco para a relação pais-bebê. Frente a isso, foram discutidas possíveis temáticas para comporem a elaboração de programas preventivos, entre elas, a relação entre o apego com a família de origem e a parentalidade, a qual pode ser definida como transição respon- 
sável por transformar o casal em pais, provocando mudanças mais profundas nas relações conjugais e na família extensa (Macarini, Crepaldi \& Vieira, 2016).

Sob este panorama, torna-se oportuno considerar os padrões relacionais de apego vivenciados na infância e os reflexos deste processo para a vida adulta, especialmente em como ele ocorre na vivência da parentalidade. Observa-se, no entanto, que a transmissão de tais padrões de apego não pode ser adotada como uma perspectiva linear, determinista e rígida, sem uma possibilidade de mudança e flexibilidade. O que os estudos contemporâneos indicam é que existe uma relação entre tais fatores, o que não significa classificar como uma relação de causa e efeito (Falcke, Wagner, \& Mosmann, 2008; Cowan \& Cowan, 2016; Semensato \& Bosa, 2013).

\subsection{Apego e o enfoque transcultural}

Até aqui pretendeu-se contextualizar de qual lugar epistemológico os autores partiram para situar a problemática do fenômeno; na sequência, os questionamentos que se fazem serão colocados no sentido de ampliar a discussão e implicação teórica ao considerar a função da cultura e a perspectiva transcultural no desenvolvimento da teoria do apego e sua interface com a parentalidade.

Ao se pensar sobre a cultura e as derivações de sua função, Nathan (1986) a define como um reservatório de significados aos quais o ser humano busca para dar sentido as suas experiências. Dessa forma, a cultura pode operar como um "envelope", propiciando segurança ao delimitar as referências de valores, códigos e crenças em que o sujeito se constituiu e está inserido; além de assumir um papel fundamental na estruturação, manutenção e transformações de sua identidade (Moro \& Lachad, 2008; Martins-Borges \& Pocreau, 2009).

Em vista disto, ressalta-se que o desafio da produção científica ao gerar novos pressupostos e teorias, esteja pautado no reconhecimento da alteridade entre os grupos sociais, acrescido do marco histórico e dos elementos da cultura que o permeiam. Assim, através da adoção de duas estratégias de investigação - a êmica e a ética, é que estão ancoradas as proposições da Psicologia transcultural (Dantas, 2012; Ueno, 2008; Ferreira, Assmar \& Souto, 2002). Na abordagem êmica procura-se analisar e compreender os comportamentos intrínsecos a contextos culturais específicos; enquanto que na vertente ética, privilegia-se a busca pela universalidade do fenômeno, e dessa forma, as teorias derivadas em um determinado contexto cultural são testadas em outros contextos (Dantas, 2012; Paiva, 2004; Berry, 1989). 
Pautada nesses princípios, a Psicologia transcultural lança um olhar que permite desenvolver teorias e métodos em determinados contextos culturais e transpõe para outros, tendo o cuidado de adaptá-los às idiossincrasias de cada grupo cultural (Ferreira, et al., 2002). Os estudos transculturais, então, surgiram a fim de generalizar a outras culturas os achados psicológicos oriundos de pesquisas realizadas na cultura norte-americana, através de estudos comparativos entre diferentes países e grupos culturais. Todavia, ao buscarem encontrar fenômenos universais, depararam-se com as diferenças intrínsecas ao contexto cultural específico; que para tanto, sua finalidade passou a constituir-se também, a compreensão do modo pelo qual as diferenças potenciais entre culturas interferem na conduta humana (Berry, Poortinga, Segall \& Dasen, 1992; Ferreira, et al., 2002).

Diante de tais fundamentos, são lançadas as seguintes indagações: a Teoria do apego pode ser utilizada em diferentes contextos culturais? Os padrões relacionais de apego, sobretudo no subsistema parental, são generalizáveis a diferentes culturas? Ou ainda, a sensibilidade materna da teoria do apego está centrada em um modelo de cuidado universal?

Respostas a tais questionamentos, não se constituem uma simples tarefa de se executar, tendo em vista correntes teóricas consolidadas que se posicionam com diferentes argumentos acerca da teoria do apego e da perspectiva transcultural. $O$ intento nesse ínterim, não é de apontar conclusões ou soluções pontuais; pelo contrário, mas o de incitar provocações teóricas que possam amplificar os constructos que já foram apresentados.

Ribas e Moura (2004) ao propor um ensaio teórico em apontar embates entre apego e cultura, destacaram pontos reflexivos que podem revisitar os fundamentos clássicos, pelos quais a teoria se constituiu e indicar diferentes olhares que permitem discutir o fenômeno. Nesse sentido, alguns autores (Van Ijzendoorn \& Sagi, 2004; Posada et al., 2002; Bretherton, 1992) ressaltam que as pesquisas realizadas por Ainsworth em Uganda, poderiam validar a universalidade da teoria do apego em termos transculturais, ao propor os diferentes estilos de apego entre participantes de culturas diversas. Todavia, tal argumento é bastante criticado por demais pesquisadores (Rothbaum Weisz, Pott, Miyake \& Morelli, 2000; Agishtein \& Brumbaugh, 2013) que afirmam sobre a aplicabilidade da teoria, sendo mais presente em populações ocidentais, especialmente em camadas de classe média e branca. Acerca disto, Fracasso, Lamb, Scholmerich e Leyendecker (1997) advertem quanto ao cuidado em legitimar os padrões de apego a um viés etnocêntrico incor- 
porando as tendências "universais ou normativas" quando replicadas em outros contextos culturais que não sejam ocidentais.

As críticas mais veiculadas à teoria do apego em diferentes culturas estão pautadas na divergência étnica entre os comportamentos de apego, tais como a noção da base segura, competência social e sensibilidade materna (Ribas, \& Moura, 2004). Isto, porque, há diferentes sistemas de cuidado implicados nas relações afetivas, especialmente nas práticas parentais (Agishtein \& Brumbaugh, 2013; McGoldrick \& Ashton, 2016; Carter \& McGoldrick, 1995).

Como exemplo, estudos de Rothbaum et al. (2000) indicam que o cuidado sensitivo e responsivo proposto por Ainsworth (1989) reflete o valor da autonomia da criança, esperado para o seu desenvolvimento na cultura ocidental. Ou seja, espera-se que o adulto aceite o bebê como uma pessoa autônoma e evite impor as suas vontades, a fim de promover o senso de autoeficácia na criança (Ribas, \& Moura, 2004). Em contraposição, a parentalidade na cultura japonesa pode ser manifesta pelo exercício da disciplina e o quanto a criança responde de forma obediente, seguindo as recomendações dadas pela família, com vistas à manutenção das heranças familiares e o respeito às hierarquias presentes (Kikuchi, 2012; Carter \& McGoldrick, 1995). Outro aspecto comum é a noção da competência social, que conforme a teoria do apego deve-se levar em conta fatores como a exploração, autonomia, manifestação espontânea do afeto e sociabilidade com as pessoas. Ao adotar esse conceito na cultura japonesa, o apego seguro não levaria em conta a sociabilidade com estranhos, uma vez que a sociabilidade não seria uma expressão de competência social nesta cultura (Ribas \& Moura, 2004).

Demais resultados empíricos (Posada et al., 2002) que avaliaram os cuidados maternos e o apego seguro entre duas cidades de países diferentes: Denver (Colorado/EUA) e Bogotá (Colômbia), apontaram que nas duas amostras de díades mãe-bebê, o apego seguro obteve a maior prevalência quando comparado ao tipo de apego inseguro (ansioso e evitativo). Entretanto, a demonstração do apego seguro apresentou-se de forma distinta entre as duas culturas: as mães colombianas expressaram maior interação com os filhos através de brincadeiras e concentraram-se em organizar um ambiente estimulante para seus bebês; enquanto que para as mães americanas, emergiu maior interação face a face e toque físico em seus bebês. Tais achados surpreenderam os pesquisadores, tendo em vista a concepção de que a cultura latina é descrita como sendo mais sociocêntrica, afetuosa e interdependente (Quezado, Costa, Peñaloza, Matos \& Ferraz, 2015; Leyendecker \& Lamb, 1999). Todavia, os autores salientam a importância de replicar tais dados em amostras maiores, 
além de considerarem que as normas de educação infantil que afetam o apego estão sob a influência da cultura.

Diante do exposto, divergentes perspectivas são discutidas acerca da implicação da teoria do apego em contextos culturais distintos, para tanto, pode-se pensar em um amadurecimento teórico capaz de integrar as dimensões psicossociais ao estabelecer padrões de apego universais que contemple adequadamente as manifestações específicas de cada cultura; o que possibilita argumentar que a presença do apego seguro, por exemplo, não significa que as crianças necessitem se comportar da mesma maneira em todas as culturas (Chao, 2001; Agishtein \& Brumbaugh, 2013; Ribas \& Moura, 2004). Com base em tais reflexões, apresenta-se como objetivo deste artigo, analisar criticamente o perfil de estudos nacionais e internacionais acerca do apego e a parentalidade sob o enfoque transcultural.

\section{MÉTODO}

\subsection{Delineamento}

Realizou-se a revisão de literatura do tipo sistemática para investigação bibliográfica. Conforme Sampaio e Mancini (2007) esse tipo de investigação visa promover uma estratégia de intervenção específica, mediante a aplicação de métodos explícitos e sistematizados de busca, apreciação crítica e síntese da informação selecionada. Ou seja, prima-se por uma técnica robusta e detalhada de avaliação que possa resumir e salientar os principais pontos da literatura investigada, por meio de critérios definidos anteriormente. As revisões sistemáticas são particularmente úteis para integrar as informações de um conjunto de estudos realizados separadamente sobre determinada intervenção, as quais podem apresentar resultados conflitantes e/ou coincidentes, bem como identificar temáticas que necessitam de evidência, auxiliando na orientação para investigações futuras.

\subsection{Procedimentos}

A revisão de literatura compreendeu as seguintes fases de elaboração: 1) Definição dos descritores e bases de dados para a revisão sistemática; 2) levantamento da produção científica em bancos de dados por meio de descritores preestabelecidos; 3) leitura dos títulos e dos resumos vinculados aos objetivos do estudo; 4) seleção dos artigos focados na temática, na íntegra; e, por fim, 5) categorização metodológica e de conteúdo das produções.

O levantamento de dados delineou-se nas seguintes bases de dados nacionais: Scielo e CAPES-Coordenação de Aperfeiçoamento de Pessoal de 
Nível Superior a base de dados internacional consultada foi a PsycINFO, de acesso restrito. Salienta-se que o acesso a base de dados Scielo ocorreu pela Biblioteca Virtual em Saúde - Psicologia Brasil (BVS-Psi); enquanto que a consulta à PsycINFO se deu pelo Portal de Periódicos CAPES/MEC.

Para a definição dos descritores optou-se pela busca na seção de terminologia em Psicologia da BVS-Psi (DeCs); enquanto que em estudos internacionais, verificou-se as palavras-chave utilizadas. Para tanto, foram considerados os estudos nos idiomas português, inglês e espanhol, entre o período de 2013 a outubro de 2017. Delimitou-se esse intervalo de tempo por contemplar o estado da arte mais recente sobre o fenômeno do apego e da parentalidade; além disso, as bases selecionadas constituem-se fontes significativas de coleta de dados em âmbito nacional e internacional, tendo em vista que a PsycINFO é sediada pela American Psychological Association (APA) e caracteriza-se por ser uma ampla base de dados em Psicologia, Educação, Psiquiatria e Ciências Sociais, vastamente utilizada em todo o mundo.

Os critérios de inclusão adotados foram: artigos empíricos entre o período de data estabelecido, com base na temática e idioma apontados; sendo excluídos os tipos de estudo em formato de capítulo de livro, livro, teses e dissertações, estudos de revisão e meta-análises. Para tal, foram escolhidos artigos indexados, os quais são submetidos a produções que passam por processo de avaliação por pares, sob controle de qualidade rigoroso. Após tais delineamentos e, com base nos descritores preestabelecidos, a combinação de estratégias de busca foram as seguintes: Apego OR Bowlby AND parentalidade OR "relação parental"; Attachment OR Bowlby AND Parenting OR Parenthood; e por fim, Apego OR Bowlby AND Parentalidad OR "relaciones parentales".

\subsection{Análise de Dados}

Após a definição dos descritores e levantamento nas bases de dados, emergiram um total de 109 artigos, os quais foram exportados para o software organizador de referências Mendeley. Posteriormente, foram excluídos estudos duplicados e que não apresentaram o texto na íntegra, restando o total de 95 trabalhos. Destes, apenas cinco estudos foram retidos; tendo em vista que a finalidade se pautou em investigar a influência da formação do apego do subsistema parental (pai e mãe) estabelecida com a família de origem e a parentalidade atual que vivencia com os filhos. Desse modo, foram excluídos estudos sobre o tipo de apego infantil e práticas parentais, apego e psicopatologia, artigos teóricos e de revisão, ensaios clínicos e estudos psicométricos sobre instrumentos que avaliem o apego. 
Na sequência, foram analisados, com base em critérios metodológicos e temáticos, os cinco artigos que estavam diretamente relacionados ao objetivo da revisão sistemática; os quais foram examinados na íntegra.

\subsection{Conflitos de interesse}

Os autores não apresentam nenhum tipo de conflitos de interesse acerca da produção realizada.

\section{RESULTADOS}

Os cinco artigos analisados nesta revisão sistemática apresentam caráter internacional, todos escritos na língua inglesa (ver Tabela 1). São estudos que, em sua maioria, foram desenvolvidos na América do Norte, mais especificamente no Canadá e nos Estados Unidos; emergindo ainda, parcerias entre pesquisadores do Vietnã e da Inglaterra (Rempel, Kuch, Rempel \& Vui, 2017; Ensink, Normandin, Plamondon, Berthelot \& Fonagy, 2016).

Durante o processo de refinamento das buscas realizadas, convém esclarecer que emergiram estudos nacionais concernentes à Teoria do apego, todavia, não foram integrados no corpus da análise, por não se constituírem o foco central de investigação. Salienta-se, portanto, que nenhum estudo nacional, entre o período e as bases estabelecidas, foi encontrado contemplando a temática sobre a formação do apego dos pais na infância e seu reflexo sobre a parentalidade.

Tabela 1.

Nome dos autores/ano, periódico, instituição e país de artigos publicados acerca do apego e parentalidade

\begin{tabular}{|c|c|c|c|}
\hline AUTORES/ANO & PERIÓDICO & INSTITUIÇÃO & PAÍS \\
\hline $\begin{array}{l}\text { 1. Rempel, Kuch, Rempel, } \\
\text { \& Vui (2017) }\end{array}$ & Developmental Psychology & $\begin{array}{c}\text { Brock University } \\
\text { St. Jerome's University } \\
\text { Hanoi National University of Education } \\
\text { Hanoi School of Public Health }\end{array}$ & Canadá e Vietnã \\
\hline $\begin{array}{l}\text { 2. Bailey, Redden, Peder- } \\
\text { son, \& Moran (2016) }\end{array}$ & $\begin{array}{c}\text { Canadian Journal of Behavioural } \\
\text { Science }\end{array}$ & $\begin{array}{l}\text { University of Guelph } \\
\text { Western University }\end{array}$ & Canadá \\
\hline $\begin{array}{l}\text { 3. Ensink, Normandin, } \\
\text { Plamondon, Fonagy \& } \\
\text { Berthelot (2016) }\end{array}$ & $\begin{array}{c}\text { Canadian Journal of Behavioural } \\
\text { Science }\end{array}$ & $\begin{array}{l}\text { Université Laval } \\
\text { Université du Québec à Trois-Rivières } \\
\text { University College London }\end{array}$ & $\begin{array}{l}\text { Canadá e } \\
\text { Inglaterra }\end{array}$ \\
\hline $\begin{array}{l}\text { 4. Madigan, Plamondon, } \\
\text { Vaillancourt, McKibbon, } \\
\text { \& Benoit (2016) }\end{array}$ & $\begin{array}{c}\text { Canadian Journal of Behavioural } \\
\text { Science }\end{array}$ & $\begin{array}{l}\text { Université Laval } \\
\text { University of Calgary } \\
\text { University of Toronto } \\
\text { Hospital for Sick Children } \\
\text { King's College London }\end{array}$ & Canadá \\
\hline $\begin{array}{l}\text { 5. Szepsenwol, Simpson, } \\
\text { Griskevicius, \& Raby } \\
\text { (2015) }\end{array}$ & $\begin{array}{c}\text { Journal of Personality and Social } \\
\text { Psychology }\end{array}$ & $\begin{array}{l}\text { University of Minnesota } \\
\text { University of Delaware }\end{array}$ & EUA \\
\hline
\end{tabular}


Quanto ao periódico que mais publicou sobre o tema, evidenciou-se o Canadian Journal of Behavioural Science, com três publicações acerca dos padrões transgeracionais do apego e a influência sobre a parentalidade/ transição para a parentalidade (Bailey, et al., 2016; Ensink et al., 2016; Madigan, et al., 2016).

No que se refere à origem dos estudos realizados, aponta-se que o Canadá obteve maior evidência que os demais citados, como os Estados Unidos, Inglaterra e Vietnã. Todavia, esse é um dado específico, tendo em vista o recorte pontual que se buscou desmembrar dentro do universo existente da Teoria do apego ao delimitar o apego e a parentalidade dos últimos cinco anos.

Na sequência, foram analisadas as características metodológicas dos artigos obtidos (ver Tabela 2), no que se refere aos dados de amostra, delineamento, tipo de estudo e técnica de coleta de dados, além de serem descritos os objetivos adotados para cada pesquisa. Em relação ao delineamento dos estudos encontrados, todos caracterizam-se pelo enfoque quantitativo. Verificou-se que dentre os cinco artigos retidos, três apresentaram como participantes mães e bebês, os quais foram denominados como díades mãe-bebê (Bailey et al., 2016; Ensink et al., 2016; Madigan et al., 2016). Nestes, a média de idade materna ficou aproximadamente na faixa dos 30 anos, com exceção do estudo de Madigan et al. (2016), por ter investigado mães adolescentes. No estudo de Szepsenwol, et al. (2015) os autores enfatizaram a diferença entre modos de cuidado parental entre mães e pais, compondo parte da amostra, 194 pais (homens), além das mães que participaram do estudo. Semelhantemente, na pesquisa de Rempel et al. (2017) foram investigados casais, cuja ênfase pautou-se também, na perspectiva da mãe e do pai sobre a formação do apego e a implicação sobre a parentalidade.

Quanto à temporalidade dos estudos analisados, constaram somente pesquisas longitudinais; as quais se derivaram em longitudinais do tipo prospectivos follow-up, que possibilitam acompanhar o fenômeno ao longo do tempo (sentido futuro). As técnicas de coleta de dados mais referidas nos artigos analisados, foram a Entrevista de Apego Adulto (AAI), seguida da Situação Estranha em pesquisas com amostras infantis. A Entrevista de Apego Adulto ou também denominada como Escala de Estilo de Relacionamento - EER), na versão traduzida, foi criada por Collins e Read (1990), os quais se utilizaram de três fatores para classificar o estilo de apego nos três tipos: seguro, inseguro evitador e inseguro ansioso. Tais fatores são explicados pelos itens de proximidade (grau de conforto com a proximidade e intimidade), confiança (grau de 
confiança e disponibilidade dos outros) e ansiedade (grau de ansiedade e medo de ser abandonado ou de não ser amado). Já a Situação Estranha, proposta por Ainsworth (1967) trata-se de um procedimento experimental que consiste em situações de ausência entre a díade mãe-bebê (de 12 a 18 meses) e posterior reencontro entre eles. Destaca-se que tal experimento objetivou comprovar a universalidade do comportamento de apego infantil frente à sinais de perigo; o que desencadeou diversos questionamentos, críticas e resultados importantes para a evolução da Teoria do Apego (van ljzendoorn \& Sagi, 2004; Posada et al., 2002; Bretherton, 1992).

Tabela 2.

Características metodológicas dos artigos analisados

\begin{tabular}{|c|c|c|c|c|c|}
\hline $\begin{array}{l}\text { AUTORES } \\
\text { (ANO) }\end{array}$ & OBJETIVOS & AMOSTRA & DELINEAMENTO & TIPO DE ESTUDO & TÉCNICA DE COLETA DE DADOS \\
\hline $\begin{array}{l}\text { 1. Rempel, Kuch, } \\
\text { Rempel \& Vui } \\
\text { (2017) }\end{array}$ & $\begin{array}{l}\text { Examinar o impacto } \\
\text { das relações positivas } \\
\text { da parentalidade sobre } \\
\text { o desenvolvimento } \\
\text { infantil, considerando } \\
\text { a formação do apego } \\
\text { entre pais e filhos. }\end{array}$ & $\begin{array}{c}802 \text { casais } \\
\text { heterossexuais }\end{array}$ & Quantitativo & Longitudinal & $\begin{array}{c}\text { Escalas: } \\
\text { - Entrevista; } \\
\text { - Father-infant relationship attitudes } \\
\text { Scale; } \\
\text { - Father-Infant } \\
\text { Interaction Scale; } \\
\text { - Self-report postnatal paternal-infant } \\
\text { attachment } \\
\text { Scale; } \\
\text { - Developmental Milestones Checklist } \\
\text { ॥ }\end{array}$ \\
\hline $\begin{array}{l}\text { 2. Bailey, Redden, } \\
\text { Pederson \& } \\
\text { Moran (2016) }\end{array}$ & $\begin{array}{l}\text { Verificar se as dificul- } \\
\text { dades relacionais entre } \\
\text { mãe e filho estariam } \\
\text { relacionadas ao tipo de } \\
\text { apego inseguro. }\end{array}$ & $\begin{array}{c}66 \text { díades } \\
\text { mãe-bebê, } \\
\text { cujos filhos } \\
\text { nasceram sem } \\
\text { complicações } \\
\text { médicas }\end{array}$ & Quantitativo & Longitudinal & $\begin{array}{c}\text { - Roteiro de observação: } \\
\text { Maternal Behaviour Q-sor; } \\
\text { Strange } \\
\text { Situation Procedure; } \\
\text { Escalas: } \\
\text { Maternal self-report measures; } \\
\text { The Depression Anxiety Stress Scales; }\end{array}$ \\
\hline $\begin{array}{l}\text { 3. Ensink, Nor- } \\
\text { mandin, Plamon- } \\
\text { don, Fonagy \& } \\
\text { Berthelot (2016) }\end{array}$ & $\begin{array}{l}\text { Analisar como se } \\
\text { constrói o apego entre } \\
\text { mães-bebês, após o } \\
\text { décimo sexto mês de } \\
\text { nascimento da criança, } \\
\text { cujas progenitoras fo- } \\
\text { ram vítimas de maltrato } \\
\text { na infância. }\end{array}$ & $\begin{array}{l}88 \text { díades } \\
\text { (mãe-bebê), } \\
\text { cujas mães } \\
\text { foram vítimas } \\
\text { de maltrato } \\
\text { infantil. }\end{array}$ & Quantitativo & Longitudinal & $\begin{array}{c}\text { Codificação do roteiro de Entrevista: } \\
\text { Reflective Functioning (RF) code of AAI } \\
\text { - Adult Attachment Interview; } \\
\text { Experimento } \\
\text { SS Procedure } \\
\text { Infant attachment security and } \\
\text { organization; } \\
\text { Experimento } \\
\text { The Sensitivity Scale }\end{array}$ \\
\hline $\begin{array}{l}\text { 4. Madigan, } \\
\text { Plamondon, } \\
\text { Vaillancourt, Mc- } \\
\text { Kibbon \& Benoit } \\
\text { (2016) }\end{array}$ & $\begin{array}{l}\text { Verificar as relações } \\
\text { entre a classificação } \\
\text { de perdas e trauma } \\
\text { não resolvidas durante } \\
\text { a transição para a } \\
\text { parentalidade, em uma } \\
\text { amostra de mães ado- } \\
\text { lescentes de alto risco. }\end{array}$ & $\begin{array}{c}55 \text { adolescen- } \\
\text { tes grávidas }\end{array}$ & Quantitativo & Longitudinal & $\begin{array}{c}\text { Codificação da entrevista de apego: } \\
\text { Adult Attachment Interview; } \\
\text { Questionário CTQ- subescala da } \\
\text { Emotional Abuse, Physical Abuse, } \\
\text { Sexual Abuse, Physical } \\
\text { Neglect, and Emotional Neglect; } \\
\text { Questionário A-DES - Self-report of } \\
\text { normal and pathological dissociative } \\
\text { experiences in adolescents; }\end{array}$ \\
\hline
\end{tabular}




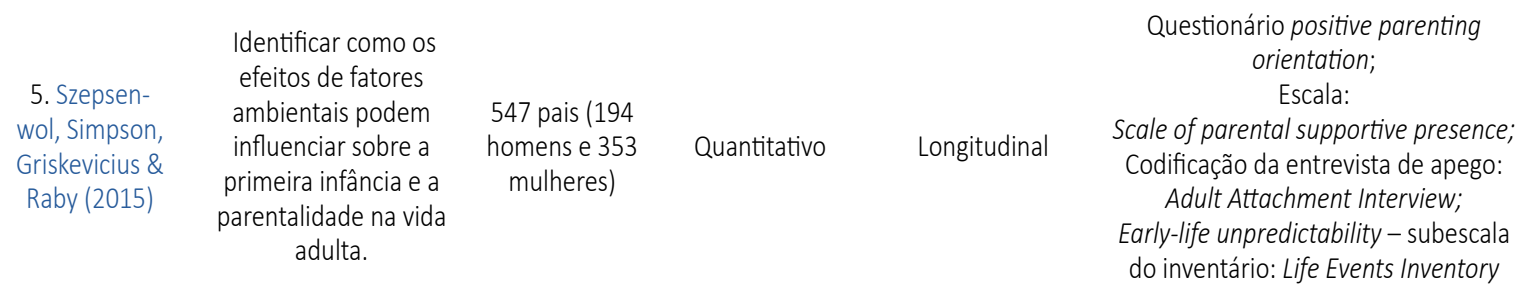

Com base nas análises metodológicas discutidas, definiram-se duas categorias temáticas que contêm discussões gerais acerca dos objetivos (ver tabela 2) apresentados nos cinco artigos, a saber: Fatores que influenciam a formação do apego e a parentalidade e Apego e relações parentais. Tais categorias serão exibidas e discutidas, a seguir.

\subsection{Fatores que influenciam a formação do apego e a parentalidade}

Entre os estudos analisados, três referiram-se à fatores de risco vivenciados na infância com a família de origem e a repercussão de tais vivências para a vida adulta, especialmente na transição para a parentalidade (Ensink et al, 2016; Madigan et al., 2016; Szepsenwol, et al., 2015). Os três estudos apontaram de modo unânime que a ausência ou restrito suporte materno constituíram-se aspectos presentes na vida dos participantes, os quais por meio de instrumentos que avaliaram o apego infantil retrospectivo, e com destaque, à Entrevista de Apego adulto, apresentaram maiores dificuldades no âmbito de relação parental ao se tornarem pais.

Na pesquisa de Ensink et al. (2016) as 88 díades mãe-bebê, cujas progenitoras foram vítimas de maltrato na infância, apresentaram maiores associações de comportamentos parentais negativos, tais como desinteresse e irritabilidade com os filhos. Sobre isto, Berthelot, et al. (2015) discutem que mães que sofreram traumas no passado podem estar mais propensas a desenvolver o tipo de apego desorganizado com seus filhos; o que apareceu em uma amostra em que todas as mães tiveram histórias de abuso e negligência no estudo desses autores.

Por sua vez, o trabalho de Szepsenwol et al. (2015) trouxe a discussão sobre diferentes fatores de risco implicados para a parentalidade, além da dimensão afetiva nos relacionamentos. Apontou que o baixo nível socioeconômico e uma rede social significativa frágil também se constituíram aspectos que contribuíram para o menor envolvimento paterno, estabelecendo relações com o estilo de apego inseguro e evitador com os filhos. Esta pesquisa corrobora com as duas anteriores ao destacar que a privação materna na 
infância e relações de baixo suporte parental foram fatores expressivos para o desenvolvimento pessoal e parental dos participantes.

Em termos gerais, aponta-se que a presente categoria de fatores que influenciam a formação do apego, levando em consideração a transmissão intergeracional e a vivência da parentalidade, discute aspectos semelhantes em diferentes dados amostrais. Nesse sentido, os estudos apresentaram amostras bem variadas no que tange à etnia e os dados sociodemográficos de ocupação laboral, arranjo familiar e escolaridade. Foram pesquisados pais e mães de ascendência africana, americana, portuguesa, hispânica, canadense e asiática. Apenas o estudo de Ensink et al. (2016) contemplou uma amostra total de caucasianos, uma vez que a maior parte da composição étnica Canadense de onde foram coletados os dados, emerge da França.

\subsection{Apego e relações parentais}

Esta categoria temática contemplou dois artigos (Rempel et al., 2017; Bailey et al., 2016) que apresentam relatos sobre a vivência da parentalidade, a partir do tipo de apego estabelecido entre pais (pai/mãe) e filhos. Os resultados apontaram diferentes modos de envolvimento e padrões relacionais entre os progrenitores e seus filhos. Apontamentos sobre estilos de apego e contextos que promovem a saúde foram apresentados, conforme se discute, a seguir.

A pesquisa de Rempel et al. (2017) trouxe importantes contribuições a respeito do envolvimento paterno, cultura e a promoção do apego na família. A amostra de 802 casais heterossexuais distribuídos entre um grupo controle e um grupo experimental, apresentaram perspectivas saudáveis e positivas da parentalidade. Foram investigados pais e mães Vietnameses, cujo grupo experimental foi submetido a intervenções de um grupo de pais no período pré-natal e, posteriormente, foram submetidos a avaliações sobre apego e envolvimento ao primeiro, quarto e nono mês de nascimento do bebê. A intervenção possibilitou aos pais, refletirem sobre a sua própria história e desenvolverem novas reflexões acerca do que é ser pai e mãe; cuidados parentais e expectativas sobre a transição para a parentalidade. Já o grupo controle consistiu em uma amostra de 12 casais heterossexuais vietnameses de uma comunidade próxima à Hanoi. Os resultados demonstraram que, embora, a história de vida paterna nem sempre pôde alcançar os efeitos esperados, ao apego com os filhos foi incentivado e os pais puderam ser encorajados a desenvolver relações positivas com seus filhos, especialmente na infância. 
Alguns dados culturais interessantes que emergiram na pesquisa de Rempel et al. (2017), foi o fato de que no grupo experimental, surpreendentemente nessa cultura, os pais (homens) apresentaram um forte vínculo afetivo com as filhas meninas; tendo em vista que no Vietnã, ainda na contemporaneidade, a preferência e o maior envolvimento paterno ocorre com filhos meninos. É possível refletir que o espaço grupal pode promover trocas saudáveis entre seus membros quanto aos padrões de enxergar os valores culturais e também, de poder questioná-los e mostrar-se receptivo às mudanças.

Já no artigo de Bailey et al. (2016) cuja amostra foi composta por 66 díades mãe-bebê canadenses, apresentou-se outro espectro da vivência da parentalidade, a partir das influências recebidas na infância quanto às representações afetivas. Nessa pesquisa foram evidenciadas dificuldades relacionais entre o estabelecimento dos vínculos afetivos mãe-bebê. Como esperado, a sensibilidade materna foi associada a maior proximidade e apego seguro; todavia, os resultados evidenciaram díades evitativas, caracterizadas pela incongruência entre o auto-relato materno e o comportamento observado das mães com seus filhos. Foram apresentados menor envolvimento e sensibilidade materna, cujos bebês demonstraram maior resistência e evitação ao permanecerem com a mãe no experimento da Situação Estranha realizado. Denota-se a importância em clarificar aspectos que norteiam a sensibilidade materna e vislumbrar o constructo através de um enfoque que considere as diferentes expressões da cultura.

\section{DISCUSSÃO}

Os artigos retidos para análise apresentam, na íntegra, caráter internacional, sendo escritos na língua inglesa. Conforme a vasta revisão de literatura sistemática proposta por Gomes e Melchiori (2012), a qual contemplou obras nacionais e internacionais do tipo: artigos indexados, livros, capítulos de livros, teses, dissertações nos idiomas português, inglês, espanhol, italiano, francês e alemão, verificou-se que de modo geral, quase todos os trabalhos obtidos sobre o apego são escritos em inglês. Para tanto, as autoras justificam que não apenas, em virtude de a língua inglesa ser a língua mãe de autores concentrados em instituições norte-americanas e inglesas, mas, especialmente, porque pesquisadores de outras nacionalidades, tais como a Holanda e Israel, tendem a divulgar seus artigos nesse idioma.

Evidenciou-se que o Canadian Journal of Behavioural Science, foi o periódico que mais publicou sobre o tema, cujas publicações pautaram-se acerca dos padrões transgeracionais do apego e a influência sobre a parentalidade/ 
transição para a parentalidade (Bailey, et al., 2016; Ensink et al., 2016; Madigan, et al., 2016). De acordo com a APA (2017), o periódico Canadian Journal of Behavioural Science apresentou no ano de 2016 uma edição especial acerca do desenvolvimento da Teoria do apego, o que parece estar relacionado às três publicações na presente revista consoante à temática.

Na revisão realizada, o país que mais apresentou investigações acerca da teoria do Apego, nos últimos cinco anos, foi o Canadá. Acerca disto, Gomes e Melchiori (2012) afirmam que grande parte dos estudiosos sobre a presente Teoria estão concentrados na América do Norte, seguidamente da Inglaterra. Por tal motivo, as universidades que mais se sobressaíram nessa revisão foram universidades canadenses, tais como a Université Laval Université du Québec à Trois-Rivières e a Brock University.

Os estudos analisados demonstraram diferentes arranjos familiares entre os participantes. Em vista disto, alguns autores (Matos, Magalhães, FéresCarneiro \& Machado, 2017; Bueno \& Vieira, 2014; Dessen \& Ribeiro, 2013; Bossardi, Gomes, Vieira \& Crepaldi, 2013) refletem sobre o maior envolvimento paterno dentro da dinâmica familiar nas últimas décadas, cujos papéis sociais atribuídos a homens e mulheres estão em constante transformação. Assim, Bossardi et al. (2013) em uma revisão sistemática, apontam sobre a crescente produção de estudos científicos que têm contemplado o fenômeno do envolvimento paterno e, deste modo, indicam pesquisas que têm incluído a participação de pais (homens) na composição amostral e discussão de seus estudos.

O delineamento quantitativo foi homogêneo entre todos os estudos encontrados. Tais achados também foram encontrados na revisão proposta por Gomes e Melchiori (2012), cujo predomínio de estudos desenvolvidos também apresentaram abordagem quantitativa e análises estatísticas. Embora a maioria das pesquisas deste trabalho, tenham mencionado utilizar-se de roteiros de entrevistas semi-estruturadas, as mesmas foram codificadas por frequência e, na sequência, classificadas em escalas para análises estatísticas posteriores. De acordo com Sampieri, Collado e Lucio (2013), o método quantitativo se utiliza da coleta e análise de dados para responder às questões de pesquisa e testar as hipóteses estabelecidas previamente; desta forma, com base na medição numérica, contagem e frequentemente pelo uso de estatística, visa-se estabelecer com exatidão os padrões de comportamento de uma população. Entretanto, seria interessante o desenvolvimento 
de estudos qualitativos e/ou mistos, a fim de integrar diferentes recursos metodológicos para acessar e vislumbrar o fenômeno investigado em uma perspectiva mais ampla e profunda dos dados, o que permitiria maior teorização, exploração e diversidade dos achados (Creswell, 2010; Sampieri et al., 2013).

Em relação à temporalidade dos métodos empregados, todas as pesquisas caracterizaram-se como longitudinais. Conforme Hochman, Nahas, Oliveira Filho e Ferreira (2005), pesquisas longitudinais apresentam grandes contribuições para o avanço do conhecimento decorrente da possibilidade de acompanhar as mudanças ocorridas ao longo do tempo; todavia, Araujo (2016) salienta a dificuldade de se realizarem estudos longitudinais em âmbito nacional, dado o grande porte e custo elevado, pela variação dos valores de referência de diferentes países e utilização de diversos métodos de pesquisa para a implantação de tais projetos. Na revisão realizada, o tempo de acompanhamento no follow-up variou entre 9 meses e 3 anos e cinco meses.

Gomes e Melchiori (2012) também constataram na revisão de literatura, que todos os estudos empíricos realizados sobre o apego utilizaram diferentes instrumentos, especialmente psicométricos, para analisar as diferentes variáveis envolvidas. Tal constatação também foi encontrada nessa revisão sistemática, tendo em vista que nenhum estudo se utilizou de um único instrumento para análise; pelo contrário, verificou-se em todos os artigos a utilização de diversas modalidades de avaliação, tais como escalas, questionários, experimentos, roteiro de observação e entrevistas.

A categoria temática que contemplou os fatores influenciadores na formação do apego e a parentalidade, apontou sobre uma variedade de fatores contextuais e culturais, cujas pesquisas foram desenvolvidas sob a ótica da Teoria do apego. Em conformidade com a discussão inicial desse artigo acerca do apego sob o enfoque transcultural, os três estudos da presente categoria não apresentaram dissonâncias teóricas quanto aos tipos de cuidados parentais e estilos de apego propostos por Ainsworth (1967). Pode-se pensar que, embora os participantes do estudo tivessem ascendências variadas, as amostras pesquisadas eram de homens e mulheres que vivem em países norte-americanos, e, portanto, ocidentais. Desse modo, padrões culturais muito distintos não foram observados. 
Os estudos foram unânimes em discutir sobre o prejuízo dos fatores de risco vivenciados pelos progenitores na infância e a repercussão de tais vivências na vida adulta, especialmente na experiência da parentalidade. Destaca-se que esses fatores podem interferir ainda, sobre o desenvolvimento infantil, como as características interpessoais dos pais, tais como grau de depressão, nível de escolaridade, experiências intergeraionais aprendidas sobre cuidados parentais, qualidade do relacionamento conjugal e as redes de apoio social. Não obstante, famílias com ausência de um dos pais ou com padrões abusivos de relacionamento e/ou abuso de drogas, também se caracterizam como fatores de risco que dificultam o desenvolvimento da criança (Grunspun, 2008; Portes, Vieira, Crepaldi, Moré \& Motta, 2013).

Os resultados encontrados nessa revisão, no que se refere à negligência materna, mães que foram vítimas de violência na infância, rede social frágil e baixo nível socioeconômico e de suporte parental, cujos fatores influenciaram na formação do apego e a parentalidade, também foram encontrados, de forma semelhante, no estudo de Madigan et al. (2016). Ao investigar as relações entre perdas e traumas de 55 mães adolescentes e a transição para a parentalidade, verificou-se que desde o pré-natal até os seis e 12 meses de vida do bebê, essa transição no ciclo vida pessoal e familiar foi marcada por dificuldades relacionais na díade mãe-filho, tendo em vista que as memórias traumáticas, especialmente pela perda da figura materna quando eram crianças/jovens, tornou a maternidade mais difícil de ser vivenciada. Portanto, verificou-se maiores prevalências de apego inseguro do tipo ansioso nas mães, especialmente após a parentalidade. No quesito traumático que emergiu na história de vida das mães adolescentes, faz-se alusão ao abuso de violência física e sexual (Madigan et al., 2016).

A segunda categoria temática, a qual se tratou do apego e das relações parentais, emergiram-se diferentes panoramas entre os achados de Rempel et al. (2017) e Bailey et al. (2016). Com base nos resultados observados, entre a experiência de fatores positivos e negativos no desenvolvimento do apego na parentalidade, é importante destacar formas de prevenção à violência no ambiente familiar e aspectos que promovam o bem-estar relacional entre seus membros. Frente à tais achados, Altafim (2017) reflete que a realização de programas de intervenção para pais pode atuar como um mecanismo de proteção, modificando os efeitos adversos dos fatores de risco que podem influenciar no desenvolvimento da criança. Além disso, aponta-se a necessidade da verificação de adequação cultural, de programas parentais desenvolvidos em diferentes países; e, nesse aspecto, cita-se a Teoria do apego, 
que conforme já argumentado, torna-se fundante apresentar um olhar que considere as variações culturais quanto ao que se concebe por sistemas de cuidados parentais e se busque uma perspectiva mais ampliada sobre os estilos de apego.

\section{CONSIDERAÇÕES FINAIS}

Esse artigo buscou analisar sistematicamente a produção científica acerca do apego e a parentalidade sob o enfoque transcultural. Para tanto, inicialmente se propôs a discutir eixos centrais que norteiam a Teoria do apego e, posteriormente, relacioná-los com provocações teóricas subjacentes à Psicologia transcultural e às dimensões psicossociais que envolvem a temática. Por fim, realizou-se uma revisão de literatura sistemática em bancos de dados nacionais e internacionais com uma matriz de análise e descritores preestabelecidos, os quais foram discutidos com base nas proposições iniciais.

Apontaram-se lacunas existentes no que se refere à produção científica especificamente ao tema elencado; tendo em vista a diversidade de estudos que englobam os sistemas de apego e temas transversais, como apego e desenvolvimento infantil, apego e psicopatologia, apego e maternidade, entre outros. Todavia, poucos artigos que contemplam o apego e a parentalidade em uma perspectiva intergeracional, foram encontrados. Acerca disto é possível sugerir o incremento de novos estudos que contemplem tal temática, considerando também a aplicabilidade da teoria à diferentes contextos culturais, históricos e sociais. Como exemplo, aponta-se a lacuna na produção científica e empírica do apego e a parentalidade no cenário latino-americano; o que caracteriza um avanço importante na área.

Além disso, torna-se necessário destacar alguns limites encontrados no desenvolvimento deste estudo, para tal, menciona-se a restrição das bases de dados e os descritores estabelecidos. É possível que com a utilização de diferentes estratégias de busca e a procura por outras bases de dados, novos resultados sejam obtidos; nesse sentido, sugerem-se revisões que possam ampliar os idiomas definidos e o intervalo de tempo para os últimos 10 anos, haja vista a especificidade do tema. Outro limitador encontrado consistiu na exclusão de outros tipos de obra, como teses e dissertações, monografias, livros e capítulos de livro, os quais também poderiam agregar informações e achados importantes sobre a temática.

Salienta-se ainda, acerca da Teoria do apego e suas implicações em pesquisas; que a mesma deve estar aberta para revisão e a consideração das 
dimensões relacionadas à cultura. Tal posicionamento não significa desprender-se da teoria e sua metodologia, mas ajustá-la para outros contextos e condições culturais. Ademais, na revisão realizada não foram encontrados estudos transculturais que comparassem diferentes culturas sobre o apego e a parentalidade numa perspectiva intergeracional do apego; somente estudos aplicados em populações locais, cujos participantes tinham ascendência de diversas nacionalidades. Por conseguinte, sugere-se o avanço para as próximas pesquisas na área. Em especial, aponta-se para o incremento de estudos que promovam reflexos para a prática profissional do psicólogo e o fomento de programas e políticas públicas destinadas ao contexto nacional, os quais venham disseminar intervenções parentais positivas para o ciclo de vida pessoal e familiar dos sujeitos.

Financiamiento: Bolsa de Pesquisa/Doutorado: CAPES

\section{REFERÊNCIAS}

Agishtein, P., \& Brumbaugh, C. (2013). Cultural variation in adult attachment: the impact of ethnicity, collectivism, and country of origin. Journal of Social, Evolutionary, and Cultural Psychology, 7(4), 384-405. https://doi.org/10.1037/ h0099181

Ainsworth, M.D.S. (1967). Infancy in Uganda: infant care and growth of love. Baltimore: John Hopkins.

Ainsworth, M. D. S. (1989). Attachments beyond infancy. American Psychologist, 44, 709-716. https://doi.org/10.1037/0003-066X.44.4.709.

Altafim, E. R. P. (2017). Avaliação da eficácia de um programa de intervenção preventiva em práticas educativas parentais. (Tese de doutorado). Universidade de São Paulo, São Paulo, Brasil.

APA (2017). Attachment Theory and Developmental Psychology/Théorie de l'attachement et psychologie du développement. Acessado em 07 de dezembro de 2017. Disponível em: http://www.apa.org/pubs/journals/cbs/?tab=5

Araujo, M. L. D. (2016). Alterações antropométricas e metabólicas: um estudo longitudinal em adolescentes do sexo feminino de Recife/PE. (Tese de Doutorado). Universidade Federal de Pernambuco, Recife, Brasil.

Arcón, V. A. B., \& Sánchez, A. R. R. (2018). Salud mental como efecto del desarrollo psicoafectivo en la infância. Psicogente, 21(39), 183-202. Disponível em: http:// www.scielo.org.co/pdf/psico/v21n39/0124-0137-psico-21-39-00183.pdf

Backes, M. S. (2018). A relação entre o envolvimento paterno e a abertura ao mundo em pais de crianças entre quatro a seis anos (Tese de Doutorado em Psicologia). Universidade Federal de Santa Catarina, Florianópolis, Brasil.

Bailey, H.N., Redden, E., Pederson, D. E., \& Moran, G. (2016). Parental Disavowal of Relationship Difficulties Fosters the Development of Insecure Attachment. Canadian Journal of Behavioural Science, 48(1), 49-59. https://doi.org/10.1037/cbs0000033

Berry, J.W. (1989). Impedes etics, emics and derived etics: The operationalization of a compelling idea. International Journal of Psychology, 24, 721-735. https://doi. org/10.1080/00207598908247841 
Berry, J. W., Poortinga, Y. H., Segall, M. H. \& Dasen, P. R. (1992). Cross-cultural psychology: Research and applications. New York: Cambridge University Press.

Berthelot, N., Ensink, K., Bernazzani, O., Normandin, L., Luyten, P., \& Fonagy, P. (2015). Intergenerational transmission of attachment in abused and neglected mothers: The role of trauma-specific reflective functioning. Infant Mental Health Journal, 36, 200-212. https://doi.org/10.1002/imhj.21499

Bolsoni-Silva, A. T., Rodrigues, O. M. P. R., Abramides, D. V. M., Souza, L. S., \& Loureiro, S. R. (2007). Práticas educativas parentais de crianças com deficiência auditiva e de linguagem. Revista Brasileira de Educação Especial, 16(2), 265-282. https://doi.org/10.1590/S1413-65382010000200008

Bretherton, I. (1992). The origins of attachment theory: John Bowlby and Mary Ainsworth. Developmental Psychology, 28, 759-775. https://doi.org/10.1037/0012-1649.28.5.759

Bolze, S. D. A. (2016). Táticas de resolução de conflitos conjugais e parentais: Uma perspectiva da transmissão intergeracional (Tese de Doutorado em Psicologia). Universidade Federal de Santa Catarina, Florianópolis, Brasil. Disponível em https://repositorio.ufsc.br/bitstream/handle/123456789/177760/346987. pdf?sequence $=1$

Bossardi, C. N., Gomes, L., Vieira, M. L., \& Crepaldi, M. A. (2013). Engajamento paterno no cuidado a crianças de 4 a 6 anos. Psicologia Argumento, 31(73), 237-246. Disponível em: https://periodicos.pucpr.br/index.php/psicologiaargumento/ article/view/20267/pdf

Bowlby, J. (1988). A secure base: parent-child attachment and healthy human development. New York: Basic Books.

Bueno, R. V., \& Vieira, M. L. (2014). Análise de estudos brasileiros sobre o pai e o desenvolvimento infantil. Psicologia Argumento, 32(76), 151-159. https://doi.org/10.7213/psicol.argum.32.076.AO10

Byng-Hall, J. (1995). Rewriting Family scripts: improvisations and systems change. New York/London: The Guilford Press.

Carter, B., \& McGoldrick, M. (1995). Etnicidade e o ciclo de vida familiar. In B. Carter, \& M. McGoldrick, As mudanças no ciclo de vida familiar: Uma estrutura para a terapia familiar (pp.65-82). Porto Alegre: Artmed.

Chao, R. (2001). Integrating culture and attachment. American Psychologist, 56, 822823. https://doi.org/10.1037/0003-066X.56.10.822

Cia, F., Pamplin, R. C. de O., \& Williams, L. C. de A. (2008). O impacto do envolvimento parental no desempenho acadêmico de crianças escolares. Psicologia em Estudo, 13(2), 351-360. https://doi.org/10.1590/S1413-73722008000200018

Collins, N. L., \& Read, S. J. (1990). Adult attachment, working models, and relationship quality in dating couples. Journal of Personality and Social Psychology, 58, 644-663. https://doi.org/10.1037/0022-3514.58.4.644

Cowan, P. A., \& Cowan, C. P. (2016). Transições familiares normativas, qualidade da relação do casal e desenvolvimento sadio dos filhos. In F. Walsh (Org.), Processos normativos da familia - Diversidade e Complexidade. Porto Alegre: Artmed.

Creswell, J. W. (2010). Projeto de pesquisa: Métodos qualitativo, quantitativo e misto. Porto Alegre: Artmed.

Crowell, J., \& Treboux, D. (2001). Attachment security in adult partnerships. In C. Clulow (Org.), Adult attachment and couple psychotherapy (pp. 28-42). London/ New York: Routledge.

Dantas, S. D. (2012). Saúde mental e interculturalidade: Implicações e novas proposições diante dos desafios em tempos de globalização. In S. D. Dantas, 
(Ogrs.), Diálogos Interculturais: Reflexões Interdisciplinares e Intervenções Psicossociais (pp. 109-160). São Paulo: Instituto de Estudos Avançados da Universidade de São Paulo.

Dessen, M. A. \& Ribeiro, M. O. (2013). Envolvimento Paterno Durante o Nascimento dos Filhos: Pai "Real" e "Ideal" na Perspectiva Materna. Psicologia: Reflexão e Crítica, 26(1), 184-192. https://doi.org/10.1590/S0102-79722013000100020

Ensink, K., Normandin, L., Plamondon, A., Fonagy, P., \& Berthelot, N. (2016). Intergenerational Pathways from Reflective Functioning to Infant Attachment Through Parenting. Canadian Journal of Behavioural Science, 48(1), 9-18. https://doi.org/10.1037/cbs0000030

Falcke, D., Wagner, A., \& Mosmann, C. P. (2008). The Relationship Between Family of Origin and Marital Adjustment for Couples in Brazil. Journal of Family Psychotherapy, 19, 170-186. https://doi.org/10.1080/08975350801905020

Ferreira, M. C., Assmar, E. M. L., \& Souto, S. O. (2002). O individualismo e - coletivismo como indicadores de culturas nacionais: convergências e divergências teórico-metodológicas. Psicologia em Estudo, 7(1), 81-89. https://doi.org/10.1590/\$1413-73722002000100011

Fracasso, M. P., Lamb, M. E., Scholmerich, A., \& Leyendecker, B. (1997). The ecology of mother-infant interaction in Euro-American and immigrant Central American families living in the United States. International Journal of Behavioral Development, 20, 207-217. https://doi.org/10.1080/016502597385298

Gomes, V. F., \& Bosa, C. A. (2010). Representações mentais de apego e percepção de práticas parentais por jovens adultas. Psicologia: Reflexão e Crítica, 23(1), 11-18. https://doi.org/10.1590/S0102-79722010000100003

Gomes, A. de A. \& Melchiori, L.E. (2012). A teoria do Apego no contexto da produção científica. São Paulo: Cultura Acadêmica

Grunspum, H. (2008). A criança resiliente - resiliência e vulnerabilidade na criança. In: F.B. Assumpção Junior, E. Kuczynski, Situações psicossociais na infância e na adolescência, pp.59-90. São Paulo: Atheneu.

Hochman, B., Nahas, F. X., Oliveira Filho, R. S. de, \& Ferreira, L. M. (2005). Desenhos de pesquisa. Acta Cirurgica Brasileira, 20(Suppl. 2), 2-9. https://dx.doi.org/10.1590/ S0102-86502005000800002

Hauser, S. (2007). Das verlorene Selbst: auswirkungen früher Verlusterfahrungen auf die adoleszente Entwicklung des Selbst. Selbstpsychologie: Europäische Zeitschrift für psychoanalytische Therapieund Forschung, 8(28), 147-73.

Hazan, C., \& Shaver, P. R. (1994). Attachment as an organizational framework for research on close relationships. Psychological Inquiry, 5, 1-22. https://doi.org/10.1207/s15327965pli0501_1

Johnson, S. M., \& Whifen, V. E. (2012). Os processos do apego na terapia de casal e família. São Paulo: Roca.

Juric, S. (2009). The connection between adult partner attachment and parenting styles. Horizons of Psychology, 18(1), 63-87. Disponível em: https://www. researchgate.net/publication/282476505_Attachment_and_Parenting_Styles

Kikuchi, W. (2012). Relações hierárquicas do Japão contemporâneo: um estudo da consciência de hierarquia na sociedade japonea (Tese de Doutorado em Sociologia). Universidade de São Paulo, São Paulo, Brasil.

Leyendecker, B., \& Lamb, M. E. (1999). Latino families. In M. E. Lamb (Ed.), Parenting and child development in "nontraditional" families (pp. 247-262). Mahwah, NJ, US: Lawrence Erlbaum Associates Publishers. 
Leyendecker, B., Lamb, M. E., \& Scholmerich, A., \& Fricke, D. M. (1997). Contexts as moderators of observed interactions: A study of Costa Rican mothers and infants from differing socioeconomic backgrounds. International Journal of Behavioral Development, 21, 15-34. https://doi.org/10.1080/016502597384965

Macarini, S. M., Crepaldi, M. A., \& Vieira, M. L. (2016). A questão da parentalidade: Contribuições para o trabalho do psicólogo na terapia de famílias com filhos pequenos. Pensando famílias, 20(2), 27-42. Disponível em: http://pepsic.bvsalud. org/scielo.php?script=sci_abstract\&pid=S1679-494X2016000200003

Madigan, S., Plamondon, A., Vaillancourt, K., McKibbon, A., \& Benoit, D. (2016). The Developmental Course of Unresolved/Disorganized States of Mind in a Sample of Adolescents Transitioning Into Parenthood. Canadian Journal of Behavioural Science, 48,(1), 19-31. https://doi.org/10.1037/cbs0000037

Matos, M. G., Magalhães, A. S., Féres-Carneiro, T., \& Machado, R. N. (2017). Construindo o Vínculo Pai-Bebê: A Experiência dos Pais. Psico-USF, 22(2), 261-271. https://doi.org/10.1590/1413-82712017220206

Martins-Borges, L. M., \& Pocreau, J. B. (2009). Reconhecer a diferença: o desafio da Etnopsiquiatria. Psicologia em Revista, 15(1), 232-245. Disponível em: http:// periodicos.pucminas.br/index.php/psicologiaemrevista/article/view/627

McGoldrick, M., \& Ashton, D. (2016). Cultura. In: F. Walsh, Processos normativos da familia: diversidade e complexidade (4a Edição). Porto Alegre: Artmed.

Melchiori, L. E., \& Dessen, M. A. (2008). A teoria do apego: contribuições para a compreensão do desenvolvimento humano. In: V. L. F. Capellini, R. M. Manzoni, (Orgs.), Políticas Públicas, práticas pedagógicas e ensino-aprendizagem: diferentes Olhares sobre o processo educacional. São Paulo: Cultura Acadêmica.

Meza, K. L., Salazar, L. D. L., \& Berrío, C. A. (2018). Relación entre apoyo familiar y el rendimiento académico en estudiantes colombianos de educación primaria. Psicogente, 21(39), 102-115. Disponível em: http://www.scielo.org.co/pdf/ psico/v21n39/0124-0137-psico-21-39-00102.pdf

Moro, M. R., \& Lachal, C. (2008). As psicoterapias - modelos, método e indicações. Petrópolis, Rio de Janeiro: Vozes.

Murta, S. G., Rodrigues, A. C., Rosa, I.de O., \& Paulo, S. G. de. (2011). Avaliação de um programa psicoeducativo de transição para a parentalidade. Paidéia (Ribeirão Preto), 22(53), 403-412. https://doi.org/10.1590/S0103-863X2012000300012

Nascimento, C. C., \& Coelho, M. R. M. (2006). Apego e perda ambígua: apontamentos para uma discussão. Revista Mal-estar e Subjetividade, 6(2), 426-449. Disponivel em: http://pepsic.bvsalud.org/scielo.php?script=sci_abstract\& pid $=$ S1518-61482006000200008

Nathan, T. (1986). La Folie des autres: Traité d'ethnopsychiatrie clinique. Paris: Dunod.

Newland, L. A., Coyl, D. D. (2010). Fathers' role as attachment figures: an interview with Sir Richard Bowlby. Early Child Development and Care, 180(1), 25-32. https://doi.org/10.1080/03004430903414679

Paiva, G. J. (2004). Aspectos da teoria do equilíbrio, de Heider, em duas subculturas étnicas brasileiras. In S. D. DeBiaggi, \& G. J. Paiva (Orgs.), Psicologia, E/Imigração e Cultura (pp. 83-96). São Paulo: Casa do Psicólogo.

Parkes, C. M. (2009). Amor e perda - As raízes do luto e suas complicações. São Paulo: Summus Editorial. 
Pereira, M. G., Ferreira, G., \& Paredes, A. C. (2012). Apego aos pais, relações românticas, estilo de vida, saúde física e mental em universitários. Psicologia: Reflexão e Crítica, 26(4), 762-771. https://doi.org/10.1590/S0102-79722013000400017

Pontes, F. A., Silva, S. S. C., Garotti, M., \& Magalhães, C. M. C. (2007). Teoria do apego: elementos para uma concepção sistêmica da vinculação humana. Aletheia, 26, 67-79. Disponível em: http://pepsic.bvsalud.org/pdf/aletheia/n26/n26a07.pdf

Portes, J. R. M., Vieira, M. L., Crepaldi, M. A., Moré, C. L. O., \& Motta, C. C. L. (2013). A criança com síndrome de Down: na perspectiva da Teoria Bioecológica do Desenvolvimento Humano, com os fatores de risco e de proteção. Boletim de Academia Paulista, 33(85), 446-464. Disponível em: http://pepsic.bvsalud.org/ scielo.php?script $=$ sci_abstract\&pid=S1415-711X2013000200015

Posada, G., Jacobs, A., Richmond, M. K., Carbonell, O. A., Alzate, G., Bustamante, M. R., \& Quiceno, J. (2002). Maternal caregiving and infant security in two cultures. Developmental Psychology, 38, 67-78. https://doi.org/10.1037/0012-1649.38.1.67

Quezado, I., Costa, J. S., Peñaloza, V., Matos, F. R., \& Ferraz, S. B. (2015). Sempre cabe mais um? Uma investigação cross-cultural do comportamento do consumidor em ambiente aglomerado. R. Adm. FACES Journal, 14(3), 56-74. Disponível em: http://www.fumec.br/revistas/facesp/article/view/2282

Reio Jr., T. G., Marcus, R. F., \& Sanders-reio, J. (2009). Contribution of student and instructor relationships and attachment style to school completion. Journal of genetic psychology, 170(1), 53-71. https://doi.org/10.3200/GNTP.170.1.53-72

Rempel, L. A., Khuc, T. N., Rempel, J., \& Vui, L. T. (2017). Influence of FatherInfant Relationship on Infant Development: A Father-Involvement Intervention in Vietnam. Developmental Psychology, 53(10), 1844-1858. https://doi. org/10.1037/dev0000390

Ribas, A. F. P., \& Moura, M. L. S. (2004). Responsividade Materna e Teoria do Apego: Uma Discussão Crítica do Papel de Estudos Transculturais. Psicologia: Reflexão e crítica, 17(3), 315-322. https://doi.org/10.1590/\$0102-79722004000300004

Rothbaum, F., Weisz, J., Pott, M., Miyake, K., \& Morelli, G. (2000). Attachment and culture: Security in the United States and Japan. American Psychologist, 55, 1093-1104. https://doi.org/10.1037/0003-066X.55.10.1093

Sampaio, R. F., \& Mancini, M. C. (2007). Estudos de revisão sistemática: um guia para síntese criteriosa da evidência científica. Revista Brasileira de Fisioterapia, 11(1), 83-89. https://doi.org/10.1590/S1413-35552007000100013

Sampieri, R. H., Collado, C. F., \& Lucio, M. P. B. (2013). Metodologia de Pesquisa. 5ạ Ed. Porto Alegre: Penso.

Schmidt, E. B., \& Argimon, I. I. L. (2009). Vinculação da gestante e apego materno fetal. Paidéia (Ribeirão Preto), 19(43), 211-220. https://doi.org/10.1590/ S0103-863X2009000200009

Scola, C., \& Vauclair, J. (2010). Infant holding side biases displayed by fathers in maternity hospitals. Journal of Reproductive and Infant Psychology, 28(1), 3-10. https://doi.org/10.1080/02646830903190938

Semensato, M. R., \& Bosa, C. A. (2013). O script de apego compartilhado no casal. Arquivos Brasileiros de Psicologia, 65(1), 138-151. Disponível em: http://pepsic. bvsalud.org/scielo.php?script=sci_arttext\&pid=S1809-52672013000100010

Szepsenwol, O., Simpson, J. A., Griskevicius, V., \& Raby, K. L. (2015). The Effect of Unpredictable Early Childhood Environments on Parenting in Adulthood. Journal 
of Personality and Social Psychology, 109(6), 1045-1067. https://doi.org/10.1037/ pspi0000032

Ueno, L. S. (2008). Migrantes em trânsito entre Brasil e Japão: Uma intervenção psicossocial no retorno. (Dissertação de Mestrado). Instituto de Psicologia da Universidade de São Paulo-USP. Disponível em: http://www.teses.usp.br/teses/ disponiveis/47/47134/tde-14012009-153211/pt-br.php

Van ljzendoorn, M., \& Sagi, A. (2004). Cultural blindness or selective inattention? American Psychologist, 56(10), 824-825. https://doi. org/10.1037/0003-066X.56.10.824

Waters, H. S., \& Waters, E. (2006). The attachment working models concept: among other things, we build script-like representations of secure base experiences. Attachment \& Human Development, 8(3), 185-197. https://doi. org/10.1080/14616730600856016 